\title{
CONSEQUENCES OF THE MARCUS/SPIELMAN/SRIVASTAVA SOLUTION OF THE KADISON-SINGER PROBLEM
}

\author{
PETER G. CASAZZA AND JANET C. TREMAIN
}

\begin{abstract}
It is known that the famous, intractible 1959 Kadison-Singer problem in $C^{*}$-algebras is equivalent to fundamental unsolved problems in a dozen areas of research in pure mathematics, applied mathematics and Engineering. The recent surprising solution to this problem by Marcus, Spielman and Srivastava was a significant achievement and a significant advance for all these areas of research. We will look at many of the known equivalent forms of the Kadison-Singer Problem and see what are the best new theorems available in each area of research as a consequence of the work of Marcus, Spielman and Srivastave. In the cases where constants are important for the theorem, we will give the best constants available in terms of a generic constant taken from [40. Thus, if better constants eventually become available, it will be simple to adapt these new constants to the theorems.
\end{abstract}

\section{INTRODUCTION}

The famous 1959 Kadison-Singer Problem [34] has defied the best efforts of some of the most talented mathematicians of the last 50 years. The recent solution to this problem by Marcus, Spielman and Srivastave [40] is not only a significant mathematical achievement by three very talented mathematicians, but it is also a major advance for a dozen different areas of research in pure mathematics, applied mathematics and engineering.

Kadison-Singer Problem (KS). Does every pure state on the (abelian) von Neumann algebra $\mathbb{D}$ of bounded diagonal operators on $\ell_{2}$ have a unique extension to a (pure) state on $B\left(\ell_{2}\right)$, the von Neumann algebra of all bounded linear operators on the Hilbert space $\ell_{2}$ ?

A state of a von Neumann algebra $\mathcal{R}$ is a linear functional $f$ on $\mathcal{R}$ for which $f(I)=1$ and $f(T) \geq 0$ whenever $T \geq 0$ (whenever $T$ is a positive operator). The set of states of $\mathcal{R}$ is a convex subset of the dual space of $\mathcal{R}$ which is

1991 Mathematics Subject Classification. Primary: 42A05,42A10,42A16,43A50,46B03, 46B07,46L05, 46L30.

The authors were supported by NSF DMS 1307685; NSF ATD 1042701 and 1321779; AFOSR DGE51: FA9550-11-1-0245. 
compact in the $\omega^{*}$-topology. By the Krein-Milman theorem, this convex set is the closed convex hull of its extreme points. The extremal elements in the space of states are called the pure states (of $\mathcal{R}$ ).

This problem arose from the very productive collaboration of Kadison and Singer in the 1950's which culminated in their seminal work on triangular operator algebras. During this collaboration, they often discussed the fundamental work of Dirac [25] on Quantum Mechanics. In particular, they kept returning to one part of Dirac's work because it seemed to be problematic. Dirac wanted to find a "representation" (an orthonormal basis) for a compatible family of observables (a commutative family of self-adjoint operators). On pages 74-75 of [25] Dirac states:

"To introduce a representation in practice

(i) We look for observables which we would like to have diagonal either because we are interested in their probabilities or for reasons of mathematical simplicity;

(ii) We must see that they all commute - a necessary condition since diagonal matrices always commute;

(iii) We then see that they form a complete commuting set, and if not we add some more commuting observables to make them into a complete commuting set;

(iv) We set up an orthogonal representation with this commuting set diagonal.

The representation is then completely determined ... by the observables that are diagonal ..."

The emphasis above was added. Dirac then talks about finding a basis that diagonalizes a self-adjoint operator, which is troublesome since there are perfectly respectable self-adjoint operators which do not have a single eigenvector. Still, there is a spectral resolution of such operators. Dirac addresses this problem on pages 57-58 of [25]:

"We have not yet considered the lengths of the basic vectors. With an orthonormal representation, the natural thing to do is to normalize the basic vectors, rather than leave their lengths arbitrary, and so introduce a further stage of simplification into the representation. However, it is possible to normalize them only if the parameters are continuous variables that can take on all values in a range, the basic vectors are eigenvectors of some observable belonging to eigenvalues in a range and are of infinite length..." 
In the case of $\mathbb{D}$, the representation is $\left\{e_{i}\right\}_{i \in I}$, the orthonormal basis of $l_{2}$. But what happens if our observables have "ranges" (intervals) in their spectra? This led Dirac to introduce his famous $\delta$-function - vectors of "infinite length." From a mathematical point of view, this is problematic. What we need is to replace the vectors $e_{i}$ by some mathematical object that is essentially the same as the vector, when there is one, but gives us something precise and usable when there is only a $\delta$-function. This leads to the "pure states" of $B\left(\ell_{2}\right)$ and, in particular, the (vector) pure states $\omega_{x}$, given by $\omega_{x}(T)=\langle T x, x\rangle$, where $x$ is a unit vector in $\mathbb{H}$. Then, $\omega_{x}(T)$ is the expectation value of $T$ in the state corresponding to $x$. This expectation is the average of values measured in the laboratory for the "observable" $T$ with the system in the state corresponding to $x$. The pure state $\omega_{e_{i}}$ can be shown to be completely determined by its values on $\mathbb{D}$; that is, each $\omega_{e_{i}}$ has a unique extension to $B\left(\ell_{2}\right)$. But there are many other pure states of $\mathbb{D}$. (The family of all pure states of $\mathbb{D}$ with the $w^{*}$-topology is $\beta(\mathbb{Z})$, the $\beta$-compactification of the integers.) Do these other pure states have unique extensions? This is the Kadison-Singer problem (KS).

By a "complete" commuting set, Dirac means what is now called a "maximal abelian self-adjoint" subalgebra of $B\left(\ell_{2}\right) ; \mathbb{D}$ is one such. There are others. For example, another is generated by an observable whose "simple" spectrum is a closed interval. Dirac's claim, in mathematical form, is that each pure state of a "complete commuting set" has a unique state extension to $B\left(\ell_{2}\right)$. Kadison and Singer show [37] that that is not so for each complete commuting set other than $\mathbb{D}$. They also show that each pure state of $\mathbb{D}$ has a unique extension to the uniform closure of the algebra of linear combinations of operators $T_{\pi}$ defined by $T_{\pi} e_{i}=e_{\pi(i)}$, where $\pi$ is a permutation of $\mathbb{Z}$.

Kadison and Singer believed that KS had a negative answer. In particular, on page 397 of [34] they state: "We incline to the view that such extension is non-unique".

Over the 55 year history of the Kadison-Singer Problem, a significant amount of research was generated resulting in a number of partial results as well as a large number of equivalent problems. These include the Anderson Paving Conjectures [2, 3, 4, the Akemann-Anderson Projection Paving Conjecture [1, the Weaver Conjectures [46, the Casazza-Tremain Conjecture [23], the Feichtinger Conjecture [14], the $R_{\epsilon}$-Conjecture [23], the Bourgain-Tzafriri Conjecture [23, the Sundberg Problem [20]. Many directions for approaching this problem were proposed and solutions were given for special cases: All matrices with positive coefficients are pavable [29] as are all matrices with "small" coefficients [11]. Under stronger hypotheses, solutions to the problem were given by Berman/Halpern/Kaftal/Weiss [8], Baranov and Dyakonov [7], Paulsen [37, 41, 42], Lata [36], Lawton [38], Popa [44], Grochenig [27], Bownik/Speegle [12], Casazza/Christensen/Lindner/Vershynin 
[14], Casazza/Christensen/Kalton [15], Casazza/Kutyniok/Speegle [21], Casazza/Edidin/Kalra/ [17] and much more.

Our goal in this paper is to see how the solution of [40 to the KadisonSinger Problem answers each of the above problems in a quantative way and to compute the best available constants at this time.

The paper is organized as follows. In Section 2 we introduce the basics of Hilbert space frame theory which forms the foundation for producing equivalences of the Paving Conjecture. Next, in Section 3 we give the basic Marcus/Spielman/Srivastava result proving Weaver's Conjecture. In Section 4 we present their proof of the Akemann-Anderson Projection Paving Conjecture and the Anderson Paving Conjecture. In Section 5 we prove the Casazza/Tremain Conjecture, the Feichtinger Conjecture, the $R_{\epsilon}$ Conjecture, and the Bourgain-Tzafriri Conjecture. In Section 6 we prove the Feichtinger Conjecture in Harmonic Analysis (the stronger form involving syndetic sets), and solve the Sundberg Problem. Section 7 contains equivalents of the Paving Conjecture for Large and Decomposable subspaces of a Hilbert space. Finally, in Section 9 we will trace some of the history of the Paving Conjecture.

\section{Frame TheOrY}

Hilbert space frame theory is the tool which is used to connect many of the equivalent forms of the Paving Conjecture. So we start with an introduction to this area. A family of vectors $\left\{f_{i}\right\}_{i \in I}$ in a Hilbert space $\mathbb{H}$ is a Riesz basic sequence if there are constants $A, B>0$ so that for all scalars $\left\{a_{i}\right\}_{i \in I}$ we have:

$$
A \sum_{i \in I}\left|a_{i}\right|^{2} \leq\left\|\sum_{i \in I} a_{i} f_{i}\right\|^{2} \leq B \sum_{i \in I}\left|a_{i}\right|^{2} .
$$

We call $A, B$ the lower and upper Riesz basis bounds for $\left\{f_{i}\right\}_{i \in I}$. If the Riesz basic sequence $\left\{f_{i}\right\}_{i \in I}$ spans $\mathbb{H}$ we call it a Riesz basis for $\mathbb{H}$. So $\left\{f_{i}\right\}_{i \in I}$ is a Riesz basis for $\mathbb{H}$ means there is an orthonormal basis $\left\{e_{i}\right\}_{i \in I}$ so that the operator $T\left(e_{i}\right)=f_{i}$ is invertible. In particular, each Riesz basis is bounded. That is, $0<\inf _{i \in I}\left\|f_{i}\right\| \leq \sup _{i \in I}\left\|f_{i}\right\|<\infty$.

Hilbert space frames were introduced by Duffin and Schaeffer [26] to address some very deep problems in nonharmonic Fourier series (see [48]). A family $\left\{f_{i}\right\}_{i \in I}$ of elements of a (finite or infinite dimensional) Hilbert space $\mathbb{H}$ is called a frame for $\mathbb{H}$ if there are constants $0<A \leq B<\infty$ (called the lower and upper frame bounds, respectively) so that for all $f \in \mathbb{H}$

$$
A\|f\|^{2} \leq \sum_{i \in I}\left|\left\langle f, f_{i}\right\rangle\right|^{2} \leq B\|f\|^{2} .
$$

If we only have the right hand inequality in Equation 2.1 we call $\left\{f_{i}\right\}_{i \in I}$ a Bessel sequence with Bessel bound B. If $A=B$, we call this an $A$-tight 
frame and if $A=B=1$, it is called a Parseval frame. If all the frame elements have the same norm, this is an equal norm frame and if the frame elements are of unit norm, it is a unit norm frame. It is immediate that $\left\|f_{i}\right\|^{2} \leq B$. If also inf $\left\|f_{i}\right\|>0,\left\{f_{i}\right\}_{i \in I}$ is a bounded frame. The numbers $\left\{\left\langle f, f_{i}\right\rangle\right\}_{i \in I}$ are the frame coefficients of the vector $f \in \mathbb{H}$. If $\left\{f_{i}\right\}_{i \in I}$ is a Bessel sequence, the synthesis operator for $\left\{f_{i}\right\}_{i \in I}$ is the bounded linear operator $T: \ell_{2}(I) \rightarrow \mathbb{H}$ given by $T\left(e_{i}\right)=f_{i}$ for all $i \in I$. The analysis operator for $\left\{f_{i}\right\}_{i \in I}$ is $T^{*}$ and satisfies: $T^{*}(f)=\sum_{i \in I}\left\langle f, f_{i}\right\rangle e_{i}$. In particular,

$$
\left\|T^{*} f\right\|^{2}=\sum_{i \in I}\left|\left\langle f, f_{i}\right\rangle\right|^{2}, \quad \text { for all } f \in \mathbb{H}
$$

and hence the smallest Bessel bound for $\left\{f_{i}\right\}_{i \in I}$ equals $\left\|T^{*}\right\|^{2}=\|T\|^{2}$. Comparing this to Equation 2.1 we have:

Theorem 2.1. Let $\mathbb{H}$ be a Hilbert space and $T: \ell_{2}(I) \rightarrow \mathbb{H}, T e_{i}=f_{i}$ be a bounded linear operator. The following are equivalent:

(1) $\left\{f_{i}\right\}_{i \in I}$ is a frame for $\mathbb{H}$.

(2) The operator $T$ is bounded, linear, and onto.

(3) The operator $T^{*}$ is an (possibly into) isomorphism.

Moreover, if $\left\{f_{i}\right\}_{i \in I}$ is a Riesz basis then it is a frame and the Riesz bounds equal the frame bounds.

It follows that a Bessel sequence is a Riesz basic sequence if and only if $T^{*}$ is onto. The frame operator for the frame is the positive, self-adjoint invertible operator $S=T T^{*}: \mathbb{H} \rightarrow \mathbb{H}$. That is,

$$
S f=T T^{*} f=T\left(\sum_{i \in I}\left\langle f, f_{i}\right\rangle e_{i}\right)=\sum_{i \in I}\left\langle f, f_{i}\right\rangle T e_{i}=\sum_{i \in I}\left\langle f, f_{i}\right\rangle f_{i}
$$

In particular,

$$
\langle S f, f\rangle=\sum_{i \in I}\left|\left\langle f, f_{i}\right\rangle\right|^{2}
$$

It follows that $\left\{f_{i}\right\}_{i \in I}$ is a frame with frame bounds $A, B$ if and only if $A \cdot I \leq$ $S \leq B \cdot I$. So $\left\{f_{i}\right\}_{i \in I}$ is a Parseval frame if and only if $S=I$. Reconstruction 
of vectors in $\mathbb{H}$ is achieved via the formula:

$$
\begin{aligned}
f & =S S^{-1} f=\sum_{i \in I}\left\langle S^{-1} f, f_{i}\right\rangle f_{i} \\
& =\sum_{i \in I}\left\langle f, S^{-1} f_{i}\right\rangle f_{i} \\
& =\sum_{i \in I}\left\langle f, f_{i}\right\rangle S^{-1} f_{i} \\
& =\sum_{i \in I}\left\langle f, S^{-1 / 2} f_{i}\right\rangle S^{-1 / 2} f_{i} .
\end{aligned}
$$

Recall that for vectors $u, v \in \mathbb{H}$ the outer product of these vectors $u v^{*}$ is the rank one operator defined by:

$$
\left(u v^{*}\right)(x)=\langle x, v\rangle u .
$$

In particular, if $\|u\|=1$ then $u u^{*}$ is the rank one projection of $\mathbb{H}$ onto span $u$. Also, $v^{*} u=\langle u, v\rangle$. The frame operator $S$ of the frame $\left\{f_{i}\right\}_{i \in I}$ is representable as

$$
S=\sum_{i \in I} f_{i} f_{i}^{*}
$$

The Gram operator of the frame $\left\{f_{i}\right\}_{i \in I}$ is $G=T^{*} T$ and has the matrix

$$
G=\left(\left\langle f_{i}, f_{j}\right\rangle\right)_{i, j \in I}=\left(f_{j}^{*} f_{i}\right)_{i, j \in I}
$$

It follows that the non-zero eigenvalues of $G$ equal the non-zero eigenvalues of $S$ and hence $\|G\|=\|S\|$.

I alsot follows that $\left\{S^{-1 / 2} f_{i}\right\}_{i \in I}$ is a Parseval frame isomorphic to $\left\{f_{i}\right\}_{i \in I}$. Two sequences $\left\{f_{i}\right\}_{i \in I}$ and $\left\{g_{i}\right\}_{i \in I}$ in a Hilbert space are isomorphic if there is a well-defined invertible operator $T$ between their spans with $T f_{i}=g_{i}$ for all $i \in I$. We now show that there is a simple way to tell when two frame sequences are isomorphic.

Proposition 2.2. Let $\left\{f_{i}\right\}_{i \in I},\left\{g_{i}\right\}_{i \in I}$ be frames for a Hilbert space $\mathbb{H}$ with analysis operators $T_{1}$ and $T_{2}$, respectively. The following are equivalent:

(1) The frames $\left\{f_{i}\right\}_{i \in I}$ and $\left\{g_{i}\right\}_{i \in I}$ are isomorphic.

(2) $\operatorname{ker} T_{1}=\operatorname{ker} T_{2}$.

Proof: $(1) \Rightarrow(2)$ : If $L f_{i}=g_{i}$ is an isomorphism, then $L f_{i}=L T_{1} e_{i}=g_{i}=$ $T_{2} e_{i}$ quickly implies our statement about kernels.

$(2) \Rightarrow(1)$ : Since $\left.\left.T_{i}\right|_{(k e r} T_{i}\right)^{\perp}$ is an isomorphism for $i=1,2$, if the kernels are equal, then

$$
T_{2}\left(\left.T_{1}\right|_{\left(k e r T_{2}\right)^{\perp}}\right)^{-1} f_{i}=g_{i}
$$

is an isomorphism. 
In the finite dimensional case, if $\left\{g_{j}\right\}_{j=1}^{n}$ is an orthonormal basis of $\ell_{2}^{n}$ consisting of eigenvectors for $S$ with respective eigenvalues $\left\{\lambda_{j}\right\}_{j=1}^{n}$, then for every $1 \leq j \leq n, \sum_{i \in I}\left|\left\langle f_{i}, g_{j}\right\rangle\right|^{2}=\lambda_{j}$. In particular, $\sum_{i \in I}\left\|f_{i}\right\|^{2}=\operatorname{trace} \mathrm{S}(=n$ if $\left\{f_{i}\right\}_{i \in I}$ is a Parseval frame). An important result is

Theorem 2.3. If $\left\{f_{i}\right\}_{i \in I}$ is a frame for $\mathbb{H}$ with frame bounds $A, B$ and $P$ is any orthogonal projection on $\mathbb{H}$, then $\left\{P f_{i}\right\}_{i \in I}$ is a frame for $P \mathbb{H}$ with frame bounds $A, B$.

Proof: For any $f \in P \mathbb{H}$,

$$
\sum_{i \in I}\left|\left\langle f, P f_{i}\right\rangle\right|^{2}=\sum_{i \in I}\left|\left\langle P f, f_{i}\right\rangle\right|^{2}=\sum_{i \in I}\left|\left\langle f, f_{i}\right\rangle\right|^{2}
$$

A fundamental result in frame theory was proved independently by Naimark and Han/Larson [24, 32]. For completeness we include its simple proof.

Theorem 2.4. A family $\left\{f_{i}\right\}_{i \in I}$ is a Parseval frame for a Hilbert space $\mathbb{H}$ if and only if there is a containing Hilbert space $\mathbb{H} \subset \ell_{2}(I)$ with an orthonormal basis $\left\{e_{i}\right\}_{i \in I}$ so that the orthogonal projection $P$ of $\ell_{2}(I)$ onto $\mathbb{H}$ satisfies $P\left(e_{i}\right)=f_{i}$ for all $i \in I$.

Proof: The "only if" part is Theorem 2.3. For the "if" part, if $\left\{f_{i}\right\}_{i \in I}$ is a Parseval frame, then the synthesis operator $T: \ell_{2}(I) \rightarrow \mathbb{H}$ is a partial isometry. So $T^{*}$ is an isometry and we can associate $\mathbb{H}$ with $T^{*} \mathbb{H}$. Now, for all $i \in I$ and all $g=T^{*} f \in T^{*} \mathbb{H}$ we have

$$
\left\langle T^{*} f, P e_{i}\right\rangle=\left\langle T^{*} f, e_{i}\right\rangle=\left\langle f, T e_{i}\right\rangle=\left\langle f, f_{i}\right\rangle=\left\langle T^{*} f, T^{*} f_{i}\right\rangle .
$$

It follows that $P e_{i}=T^{*} f_{i}$ for all $i \in I$.

For an introduction to frame theory we refer the reader to Han/Kornelson/Larson/Weber [31], Christensen [24] and Casazza/Kutyniok [20].

\section{Marcus/Spielman/Srivastave and Weaver's Conjecture}

In [40] the authors do a deep analysis of what they call mixed characteristic polynomials to prove a famous conjecture of Weaver [46] which Weaver had earlier shown is equivalent to the Paving Conjecture which Anderson [2] had previously shown was equivalent to the Kadison-Singer Problem. We will mearly state the main theorem from [40] here and use it to find the best constants in the various equivalent forms of the Kadison-Singer Problem.

Theorem 3.1 (Marcul/Spielman/Srivastava). Let $r$ be a positive integer and let $u_{1}, u_{2}, \cdots, u_{m} \in \mathbb{C}^{d}$ be vectors such that

$$
\sum_{i=1}^{m} u_{i} u_{i}^{*}=I,
$$


and $\left\|u_{i}\right\|^{2} \leq \delta$ for all $i$. Then there is a partition $\left\{S_{1}, S_{2}, \cdots, S_{r}\right\}$ of $[m]$ such that

$$
\left\|\sum_{i \in S_{j}} u_{i} u_{i}^{*}\right\| \leq\left(\frac{1}{\sqrt{r}}+\sqrt{\delta}\right)^{2} \text {, for all } j=1,2, \cdots, r .
$$

In [46], Weaver reformulated the Paving Conjecture into Discrepancy Theory which generated a number of new equivalences of the Paving Conjecture [23, 19] and set the stage for the eventual solution to the problem. Setting $r=2$ and $\delta=1 / 18$ [40], this implies the original Weaver Conjecture $K S_{2}$ [46] with $\eta=18$ and $\theta=2$.

Theorem 3.2 (Marcus/Spielman/Srivastave). There are universal constants $\eta \geq 2$ and $\theta>0$ so that the following holds. Let $u_{1}, u_{2}, \cdots, u_{m} \in \mathbb{C}^{d}$ satisfy $\left\|u_{i}\right\| \leq 1$ for all $i$ and suppose

$$
\sum_{i=1}^{M}\left|\left\langle u, u_{i}\right\rangle\right|^{2}=\eta \text {, for every unit vector } u \in \mathbb{C}^{d} .
$$

Then there is a partition $S_{1}, S_{2}$ of $\{1,2, \cdots, m\}$ so that

$$
\sum_{i \in S_{j}}\left|\left\langle u, u_{i}\right\rangle\right|^{2} \leq \eta-\theta
$$

for every unit vector $u \in \mathbb{C}^{d}$ and each $j \in\{1,2\}$.

Moreover, $\eta=18$ and $\theta=2$ work.

To make Theorem 3.1 more usable later, we will reformulate it into the language of operator theory. Recall, for a matrix operator

$$
T=\left(a_{i j}\right)_{i, j=1}^{m}
$$

we let

$$
\delta(T)=\min _{1 \leq i \leq m}\left|a_{i i}\right|
$$

Theorem 3.3. Let $r$ be a positive integer. Given an orthogonal projection $Q$ on $\ell_{2}^{m}$ with $\delta(Q) \leq \delta$, there are diagonal projections $\left\{P_{j}\right\}_{j=1}^{r}$ with

$$
\sum_{j=1}^{r} P_{j}=I
$$

and

$$
\left\|P_{j} Q P_{j}\right\| \leq\left(\frac{1}{\sqrt{r}}+\sqrt{\delta}\right)^{2} \text {, for all } j=1,2, \cdots, r \text {. }
$$


Proof. Letting $u_{i}=Q e_{i}$ for all $i=1,2, \cdots, m$, we have that $Q=\left(u_{i}^{*} u_{j}\right)_{i, j=1}^{m}$. Choose a partition $\left\{S_{j}\right\}_{j=1}^{r}$ of $[\mathrm{m}]$ satisfying Theorem 3.1 and let $P_{j}$ be the diagonal projection onto $\left\{e_{i}\right\}_{i \in S_{j}}$. For any $k \in[r]$ we have:

$$
\left\|P_{k} Q P_{k}\right\|=\left\|\left(u_{i}^{*} u_{j}\right)_{i, j \in S_{k}}\right\|=\left\|\sum_{i \in S_{k}} u_{i} u_{i}^{*}\right\| \leq\left(\frac{1}{\sqrt{r}}+\sqrt{\delta}\right)^{2} .
$$

\section{Marcus/Spielman/Srivastava and the Paving Conjectures}

Perhaps the most significant advance on the Kadison-Singer Problem occured when Anderson [2] showed that it was equivalent to what became known as the (Anderson) Paving Conjecture. The significance of this was that it removed the Kadison-Singer Problem from the burden of being a very technical problem in $C^{*}$-Algebras which had no real life outside the field, to making it a highly visible problem in Operator Theory which generated a significant amount of research - and eventually led to the solution to the problem.

Definition 4.1. Let $T: \ell_{2}^{n} \rightarrow \ell_{2}^{n}$ be an operator. Given $r \in \mathbb{N}$ and $\epsilon>0$ we say that $T$ can be $(r, \epsilon)$-paved if there is a partition $\left\{A_{j}\right\}_{j=1}^{r}$ of $[n]$ so that if $P_{i}$ is the coordinate projection onto the coordinates $A_{j}$ so that

$$
\left\|P_{i} T P_{i}\right\| \leq \epsilon\|T\|, \text { for all } i=1,2, \cdots, r .
$$

Or equivalently, there are coordinate projections $\left\{P_{i}\right\}_{i=1}^{r}$ so that

$$
\sum_{i=1}^{r} P_{i}=I \text { and }\left\|P_{i} T P_{i}\right\| \leq \epsilon\|T\| .
$$

A major advance was made on the Kadison-Singer Problem by Anderson [2].

Theorem 4.2 (Anderson Paving Conjecture). The following are equivalent:

(1) The Kadison-Singer Conjecture is true.

(2) For every $0<\epsilon<1$ there is an $r=r(\epsilon) \in \mathbb{N}$ so that every operator $T$ on a finite or infinite dimensional Hilbert space is $(r, \epsilon)$-pavable.

(3) For every $0<\epsilon<1$ there is an $r=r(\epsilon) \in \mathbb{N}$ so that every selfadjoint operator $T=T^{*}$ on a finite or infinite dimensional Hilbert space is $(r, \epsilon)$-pavable.

This result become known as the Anderson Paving Conjecture and became a major advance for the field. For the next 25 years a significant amount of effort was directed at proving (or giving a counter-example to) the Paving Conjecture. We give a brief outline of the history of this effort in Section 9.

In 1991, Akemann and Anderson reformulated the Paving Conjecture for operators into a paving conjecture for projections. They also gave a number 
of conjectures concerning paving projections and the Paving Conjecture. This was a major advance for the area reducing the problem to a very special class of operators. Theorem 3.1 also implies the Akemann-Anderson Projection Paving Conjecture [1] which they showed implies a positive solution to the Kadison-Singer Problem.

Theorem 4.3. Given $\epsilon>0$, choose $\delta>0$ so that

$$
\left(\frac{1}{\sqrt{2}}+\sqrt{\delta}\right)^{2} \leq 1-\epsilon .
$$

For any projection $Q$ on $\ell_{2}^{m}$ of rank $d$ there is a diagonal projection $P$ on $\ell_{2}^{m}$ so that

$$
\|P Q P\| \leq 1-\epsilon \text { and }\|(I-P) Q(I-P)\| \leq 1-\epsilon .
$$

Proof. This is immediate from Theorem 3.3 letting $r=2$ and noting that $P_{2}=\left(I-P_{1}\right)$.

The authors [40] then give a quantative proof of the original Anderson Paving Conjecture [2]. To do this, we will first look at an elementary way to pass between paving for operators and paving for projections introduced by Casazza/Edidin/Kalra/Paulsen [17]. In [17] there is a simple method for passing paving numbers back and forth between operators and projections with constant diagonal $1 / 2$ ( or $1 / 2^{k}$ if we iterate this result). This was a serioius change in direction for the paving conjecture for projections. The earlier work of Akemann/Anderson [1] and Weaver [46] emphasized paving for projections with small diagonal while the results in [17] showed that it is more natural to work with projections with large diagonal. The proof is a direct calculation.

Theorem 4.4 (Casazza/Edidin/Kalra/Paulsen). If $T$ is a self-adjoint operator with $\|T\| \leq 1$ then

$$
A=\left[\begin{array}{cc}
T & \sqrt{I-T^{2}} \\
\sqrt{I-T^{2}} & -T
\end{array}\right]
$$

is an idempotent. I.e. $A^{2}=I$.

Hence,

$$
P=\frac{I \pm A}{2}
$$

is a projection.

It follows that the paving numbers for self-adjoint operators are at most the square of the paving numbers for projections. Using Theorem 4.4, in [17] they prove the following (See also [40]):

Proposition 4.5 (Casazza/Edidin/Kalra/Paulsen). Suppose there is a function $r: \mathbb{R}_{+} \rightarrow \mathbb{N}$ so that every $2 n \times 2 n$ projection matrix $Q$ with diagonal 
entries equal to $1 / 2$ can be $\left(r(\epsilon), \frac{1+\epsilon}{2}\right)$-paved, for every $0<\epsilon<1$. Then every $n \times n$ self-adjoint zero diagonal matrix $T$ can be $\left(r^{2}(\epsilon), \epsilon\right)$-paved for all $0<\epsilon<1$.

Proof. Given $Q$ as in the proposition, $Q=\left(u_{i}^{*} u_{j}\right)_{i, j \in[2 n]}$ is the gram matrix of $2 n$ vectors $u_{1}, u_{2}, \cdots, u_{2 n} \in \mathbb{C}^{n}$ with $\left\|u_{i}\right\|^{2}=1 / 2=\delta$. Applying Theorem 3.3 we find a partition $\left\{A_{i}\right\}_{i=1}^{r}$ of $[2 n]$ so that if $P_{i}$ is the diagonal projection onto the coordinates of $A_{i}$ we have for $k \in[r]$,

$\left\|P_{k} T P_{k}\right\|=\left\|\left(u_{i}^{*} u_{j}\right)_{i, j \in A_{k}}\right\|=\left\|\sum_{i \in A_{k}} u_{i} u_{i}^{*}\right\| \leq\left(\frac{1}{\sqrt{r}}+\frac{1}{\sqrt{2}}\right)^{2}<\frac{1}{2}+\frac{3}{\sqrt{r}}<\frac{1+\epsilon}{2}$,

if $r=\left(\frac{6}{\epsilon}\right)^{2}$. So every $Q$ can be $\left(r, \frac{1+\epsilon}{2}\right)$-paved.

It follows that every self-adjoint operator can be $(R \epsilon)$-paved for $r=\left(\frac{6}{\epsilon}\right)^{4}$, in either the real or complex case.

Using this and Theorem [23], 40] gives a quantative proof of the Anderson Paving Conjecture and hence of the Kadison-Singer Problem.

Theorem 4.6. For every $0<\epsilon<1$, every zero-diagonal real (Resp. complex) self-adjoint matrix $T$ can be $(r, \epsilon)$-paved with $r=(6 / \epsilon)^{4}\left(\operatorname{Resp} . r=(6 / \epsilon)^{8}\right)$.

Important: For complex Hilbert spaces, given an operator $T$, we write it as $T=A+i B$ where $A, B$ are real operators. Paving $A, B$ separately and intersecting the paving sets, we have a paving of $T$ but with the paving number squared.

Remark 4.7. In [17] it is shown that $1 / \epsilon^{2} \leq r$ in Theorem 4.6. We can compare this to the value $r=\left(\frac{6}{\epsilon}\right)^{4}$ we are getting from the theorem.

\section{Equivalents of the Paving Conjecture}

Casazza/Tremain reformulated the Paving Conjecture into a number of conjectures related to problems in engineering. They also gave several conjectures related to the Paving Conjecture. Theorem 3.2 answers the CasazzaTremain Conjecture [23].

Theorem 5.1. Every unit norm 18-tight frame can be partitioned two subsets each of which has frame bounds 2, 16 .

Proof. Let $\left\{u_{i}\right\}_{i=1}^{18 d}$ be a unit norm 18-tight frame in $\mathbb{C}^{d}$. By Theorem $[3.2$, we can find a partition $\left\{S_{1}, S_{2}\right\}$ of $[18 d]$ so that for all $\|u\|=1$ we have

$$
\sum_{i \in S_{1}}\left|\left\langle u, u_{i}\right\rangle\right|^{2} \leq 16
$$


Thus,

$$
\begin{aligned}
18 & =\sum_{i \in S_{1}}\left|\left\langle u, u_{i}\right\rangle\right|^{2}+\sum_{i \in S_{2}}\left|\left\langle u, u_{i}\right\rangle\right|^{2} \\
& \leq 16+\sum_{i \in S_{2}}\left|\left\langle u, u_{i}\right\rangle\right|^{2}
\end{aligned}
$$

It follows that

$$
\sum_{i \in S_{2}}\left|\left\langle u, u_{i}\right\rangle\right|^{2} \geq 2
$$

By symmetry,

$$
\sum_{i \in S_{1}}\left|\left\langle u, u_{i}\right\rangle\right|^{2} \geq 2
$$

In his work on time-frequency analysis, Feichtinger [27, 23] noted that all of the Gabor frames he was using had the property that they could be divided into a finite number of subsets which were Riesz basic sequences. This led to a conjecture known as the Feichtinger Conjecture [14]. There is a significant body of work on this conjecture [5, 6, 27] (See also [36] for a large listing of papers on the Feichtinger Conjecture in reproducing kernel Hilbert spaces and many classical spaces such as Hardy space on the unit disk, weighted Bergman spaces, and Bargmann-Fock spaces). The following theorem gives the best quantative solution to the Feichtinger Conjecture from the results of 40 .

Theorem 5.2. Every unit norm B-Bessel sequence can be partitioned into $r$-subsets each of which is a $\epsilon$-Riesz basic sequence, where

$$
r=\left(\frac{6(B+1)}{\epsilon}\right)^{4} \text { in the real case, }
$$

and

$$
r=\left(\frac{6(B+1)}{\epsilon}\right)^{8} \text { in the complex case. }
$$

Proof. Fix $0<\epsilon<1$ and let $\left\{e_{i}\right\}_{i=1}^{\infty}$ be an orthonormal basis for $\ell_{2}$. Let $T: \ell_{2} \rightarrow \ell_{2}$ satisfy $\left\|T e_{i}\right\|=1$ for all $i=1,2, \cdots$. Let $S=T^{*} T$. Since $S$ has ones on the diagonal, $I-S$ has zero diagonal and so by Theorem 4.6 there is an

and

$$
r=\left(\frac{6(\|s\|+1)}{\epsilon}\right)^{4} \text { in the real case , }
$$

$$
r=\left(\frac{6(\|s\|+1)}{\epsilon}\right)^{8} \text { in the complex case }
$$


and a partition $\left\{S_{j}\right\}_{j=1}^{r}$ of $\mathbb{N}$ so that if $Q_{S_{j}}$ is the diagonal projection onto $\left\{e_{i}\right\}_{i \in S_{j}}$, we have

$$
\left\|Q_{S_{j}}(I-S) Q_{S_{j}}\right\| \leq \frac{\epsilon}{\|S\|+1} \|(I-S \| .
$$

Now, for all $j=1,2, \cdots, r$ and all $u=\sum_{i \in S_{j}} a_{i} e_{i}$ we have:

$$
\begin{aligned}
\left\|\sum_{i \in S_{j}} a_{i} T e_{i}\right\|^{2} & =\left\|T Q_{S_{j}} u\right\|^{2} \\
& =\left\langle T Q_{S_{j}} u, T Q_{S_{j}} u\right\rangle \\
& =\left\langle T^{*} T Q_{S_{j}} u, Q_{S_{j}} u\right\rangle \\
& =\left\langle Q_{S_{j}} u, Q_{S_{j}} u\right\rangle-\left\langle Q_{S_{j}}(I-S) Q_{S_{j}} u, Q_{S_{j}} u\right\rangle \\
& \geq\left\|Q_{S_{j}} u\right\|^{2}-\frac{\epsilon}{\|S\|+1}\|I-S\|\left\|Q_{S_{j}} u\right\|^{2} \\
& \geq(1-\epsilon)\left\|Q_{S_{j}} u\right\|^{2} \\
& =(1-\epsilon) \sum_{i \in S_{j}}\left|a_{i}\right|^{2} .
\end{aligned}
$$

Similarly,

$$
\left\|\sum_{i \in S_{j}} a_{i} T e_{i}\right\|^{2} \leq(1+\epsilon) \sum_{i \in S_{j}}\left|a_{i}\right|^{2}, \text { for all } j=1,2, \cdots, r \text {. }
$$

This result also answers the Sundberg Problem [20]. The question was: Can every bounded Bessel sequence be written as the finite union of nonspanning sets? The answer is now yes. We just partition our Bessel sequence into Riesz basic sequences. It is clear that Riesz basic sequences can be partitioned into non-spanning sets. I.e. Take one vector as one set and the rest of the vectors as the other set. Neither of these can span the Hilbert space.

This result answers another famous conjecture known as the $R_{\epsilon}$-Conjecture. This was introduced by Casazza/Vershynin (unpublished) and was shown to be equivalent to the Paving Conjecture. Recall, if $\epsilon>0$ and $\left\{u_{i}\right\}_{i=1}^{\infty}$ is a unit norm Riesz bsic sequence with Riesz bounds $A=1-\epsilon, B=1+\epsilon$ we call $\left\{u_{i}\right\}_{i \in I}$ an $\epsilon$-Riesz basic sequence. This is now a special case of the Feichtinger Conjecture, Theorem 5.2

Theorem 5.3. For every $0<\epsilon<1$ there is an $r \in \mathbb{N}$ so that every unit norm Riesz basic sequence with upper Riesz bound $B$ is a finite union of $\epsilon$-Riesz basic sequences, where $r$ is as in Theorem 5.2.

We note that Theorem 5.3 fails for equivalent norms on a Hilbert space. For example, if we renorm $\ell_{2}$ by letting $\left|\left\{a_{i}\right\}\right|=\left\|a_{i}\right\|_{\ell_{2}}+\sup _{i}\left|a_{i}\right|$, then the 
$R_{\epsilon}$-Conjecture fails for this equivalent norm. To see this, let $f_{i}=\left(e_{2 i}+\right.$ $\left.e_{2 i+1}\right) /(\sqrt{2}+1)$ where $\left\{e_{i}\right\}_{i \in \mathbb{N}}$ is the unit vector basis of $\ell_{2}$. This is now a unit norm Riesz basic sequence, but no infinite subset satisfies theorem 5.3. To check this, let $J \subset \mathbb{N}$ with $|J|=n$ and $a_{i}=1 / \sqrt{n}$ for $i \in J$. Then,

$$
\left|\sum_{i \in J} a_{i} f_{i}\right|=\frac{1}{\sqrt{2}+1}\left(\sqrt{2}+\frac{1}{\sqrt{n}}\right) .
$$

Since the norm above is bounded away from one for $n \geq 2$, we cannot satisfy the requirements of theorem 5.3 .

In 1987, Bourgain and Tzafriri 10 proved a fundamental result in Operator Theory known as the Restricted Invertibility Principle.

Theorem 5.4 (Bourgain-Tzafriri). There are universal constants $A, c>0$ so that whenever $T: \ell_{2}^{n} \rightarrow \ell_{2}^{n}$ is a linear operator for which $\left\|T e_{i}\right\|=1$, for $1 \leq i \leq n$, then there exists a subset $\sigma \subset\{1,2, \cdots, n\}$ of cardinality $|\sigma| \geq c n /\|T\|^{2}$ so that for all $j=1,2, \cdots, n$ and for all choices of scalars $\left\{a_{j}\right\}_{j \in \sigma}$,

$$
\left\|\sum_{j \in \sigma} a_{j} T e_{j}\right\|^{2} \geq A \sum_{j \in \sigma}\left|a_{j}\right|^{2}
$$

In a significant advance, Spielman and Srivastave 45] gave an algorithm for proving the restricted invertibility theorem. Theorem 5.4 gave rise to a problem in the area which has received a great deal of attention [11, 23] known as the Bourgain-Tzafriri Conjecture. No one really noticed that this result is the finite version of the Feichtinger Conjecture. This conjecture is now a theorem. The proof is identical to the proof of Theorem 5.2

Theorem 5.5. For every $0<\epsilon<1$ and for every $B>1$ there is a natural number $r$ satisfying: For any natural number $n$, if $T: \ell_{2}^{n} \rightarrow \ell_{2}^{n}$ is a linear operator with $\|T\| \leq B$ and $\left\|T e_{i}\right\|=1$ for all $i=1,2, \cdots, n$, then there is a partition $\left\{S_{j}\right\}_{j=1}^{r}$ of $\{1,2, \cdots, n\}$ so that for all $j=1,2, \cdots, r$ and all choices of scalars $\left\{a_{i}\right\}_{i \in S_{j}}$ we have:

$$
(1-\epsilon) \sum_{i \in S_{j}}\left|a_{i}\right|^{2} \leq\left\|\sum_{i \in S_{j}} a_{i} T e_{i}\right\|^{2} \leq(1+\epsilon) \sum_{i \in S_{j}}\left|a_{i}\right|^{2},
$$

where

$$
r=\left(\frac{6(B+1)}{\epsilon)}\right)^{4} \text { in the real case, }
$$

and

$$
r=\left(\frac{6(B+1)}{\epsilon}\right)^{8} \text { in the complex case }
$$




\section{Paving in Harmonic Anslysis}

Recall the definition of Laurent Operator:

Definition 6.1. If $f \in L^{\infty}[0,1]$, the Laurent operator with symbol f, denoted $M_{f}$, is the operator of multiplication by $f$.

In the 1980's, a very deep study of the Paving Conjecture for Laurant Operators was carried out by Berman/Halpern/Kaftal/Weiss [8, 9, 29]. They produced a parade of new techniques and interesting results in this direction including the introduction of the notion of uniform paving. They also showed that matrices with positive coefficients are pavable.

We need the following notation.

Notation: If $I \subset \mathbb{Z}$, we let $S(I)$ denote the $L^{2}([0,1])$-closure of the span of the exponential functions with frequencies taken from $I$ :

$$
S(I)=\operatorname{cl}\left(\operatorname{span}\left\{\mathrm{e}^{2 \pi \mathrm{i} n t}\right\}_{n \in I}\right) .
$$

A deep and fundamental question in Harmonic Analysis is to understand the distribution of the norm of a function $f \in S(I)$. It is known [8, 9, 29] if that if $[a, b] \subset[0,1]$ and $\epsilon>0$, then there is a partition of $\mathbb{Z}$ into arithmetic progressions $A_{j}=\{n r+j\}_{n \in \mathbb{Z}}, 0 \leq j \leq r-1$ so that for all $f \in S\left(A_{j}\right)$ we have

$$
(1-\epsilon)(b-a)\|f\|^{2} \leq\left\|f \cdot \chi_{[a, b]}\right\|^{2} \leq(1+\epsilon)(b-a)\|f\|^{2} .
$$

What this says is that the functions in $S\left(A_{j}\right)$ have their norms nearly uniformly distributed across $[a, b]$ and $[0,1] \backslash[a, b]$. The central question is whether such a result is true for arbitrary measurable subsets of $[0,1]$ (but it is known that the partitions can no longer be arithmetic progressions [12, 29, 30]). If $E$ is a measurable subset of $[0,1]$, let $P_{E}$ denote the orthogonal projection of $L^{2}[0,1]$ onto $L^{2}(E)$, that is, $P_{E}(f)=f \cdot \chi_{E}$. The fundamental question here for many years, is now answered by the following result which is an immediate consequence of Theorem 5.2 .

Theorem 6.2. If $E \subset[0,1]$ is measurable and $\epsilon>0$ is given, there is a partition $\left\{S_{j}\right\}_{j=1}^{r}$ of $\mathbb{Z}$ so that for all $j=1,2, \cdots, r$ and all $f \in S\left(A_{j}\right)$

$$
(1-\epsilon)|E|\|f\|^{2} \leq\left\|P_{E}(f)\right\|^{2} \leq(1+\epsilon)|E|\|f\|^{2}
$$

where

$$
r=\left(\frac{6(|E|+1)}{\epsilon|E|}\right)^{8} .
$$

Recall that $\left\{e^{2 \pi i n t}\right\}_{n \in \mathbb{Z}}$ is an orthonormal basis for $L^{2}[0,1]$. If $E \subset[0,1]$ of positive Lebesgue measure and $L^{2}(E)$ denotes the corresponding Hilbert space of square-integrable functions on $E$, then $f_{n}(t)=e^{2 \pi i n t} \chi_{E}$ for $n \in \mathbb{Z}$ is a Parseval frame for $L^{2}(E)$ called the Fourier Frame for $L^{2}(E)$. Much work 
has been expended on trying to prove the Feichtinger Conjecture for Fourier Frames.

If $f \in L^{2}[0,1]$ and $0 \neq a$ we define

$$
\left(T_{a} f\right)(t)=f(t-a) .
$$

Casazza/Christensen/Kalton [15] showed that if $f \in L^{2}[0,1]$ then $\left\{T_{n}(f)\right\}_{n \in \mathbb{N}}$ is a frame if and only if it is a Riesz basic sequence.

Halpern/Kaftal/Weiss [29] studied uniform pavings for Laurent operators. In particular, they asked when we can pave Laurent operators with arithmetic progressions from $\mathbb{Z}$ ? They showed that this occurs if and only if the symbol $f$ is Riemann Integrable. As a consequence of [40], a result of Paulsen implies that we can at least pave all Laurent operators with subsets of $\mathbb{Z}$ which have bounded gaps.

Definition 6.3. A set $S \subset \mathbb{N}$ is called syndetic if for some finite subset $F$ of $\mathbb{N}$ we have

$$
\cup_{n \in F}(S-n)=\mathbb{N}
$$

where

$$
S-n=\{m \in \mathbb{N}: m+n \in S\} .
$$

Thus syndetic sets have bounded gaps. I.e. There is an integer $p$ so that $[a, a+1, \cdots, a+p] \cap S \neq \phi$ for every $a \in \mathbb{N}$. We will call $p$ the gap length.

Paving by syndetic sets arose from the fact that the Grammian of a Fourier Frame is a Laurent matrix. Moreover, dividing frames into Riesz sequences is equivalent to paving their Grammian [42, 41, 18].

At GPOTS (2008) Paulsen presented a quite general paving result which included paving by syndetic sets. The idea was to work in $\ell^{2}(G)$ where $G$ is a countable discrete group and $G$-invariant frames - I.e. frames which are invariant under the action of $G$. Fourier frames are thus $\mathbb{Z}$-invariant. Paulsen next shows that a frame is G-invariant if and only if its Grammian belongs to the group von Neumann algebra $V N(G)$. Paulsen then shows that an element of $V N(G)$ is pavable if and only if it is pavable by syndetic sets. Unraveling the notation, it follows that $G$-invariant frames which can be partitioned into Riesz sequences can also be partitioned with syndetic partitions. These results then appeared in his paper [42, 41]. Lawton, [38] gave a direct proof of syndetic pavings for Fourier Frames. We now give this result with the constants available from [40].

Theorem 6.4. The Fourier frame $\left\{e^{2 \pi i n t} \chi_{E}\right\}_{n \in \mathbb{Z}}$ for $L^{2}(E)$ can be partitioned into $r$ syndetic sets $\left\{S_{j}\right\}_{j=1}^{r}$ with gap length $p \leq r$ so that

$$
\left\{e^{2 \pi i n t} \chi_{E}\right\}_{n \in S_{j}} \text { is a } \epsilon \text {-Riesz sequence for all } j=1,2, \cdots, r \text {, }
$$


where

$$
r=\left(\frac{6(|E|+1)}{\epsilon|E|}\right)^{8} .
$$

\section{7. "LARGE" AND "DeCOMPosable" subspaces OF $\mathbb{H}$}

In this section we give some new theorems arising from [40] relating to large and decomposable subspaces of a Hilbert space. These ideas were introduced in [19]. Throughout this section we will use the notation:

Notation: If $E \subset I$ we let $P_{E}$ denote the orthogonal projection of $\ell_{2}(I)$ onto $\ell_{2}(E)$. Also, recall that we write $\left\{e_{i}\right\}_{i \in I}$ for the standard orthonormal basis for $\ell_{2}(I)$.

For results on frames, see Section 2 ,

Definition 7.1. A subspace $\mathbb{H}$ of $\ell_{2}(I)$ is A-large for $A>0$ if it is closed and for each $i \in I$, there is a vector $f_{i} \in \mathbb{H}$ so that $\left\|f_{i}\right\|=1$ and $\left|f_{i}(i)\right| \geq A$. The space $\mathbb{H}$ is large if it is A-large for some $A>0$.

It is known that every frame is isomorphic to a Parseval frame. The next lemma gives an alternative identification of these Parseval frames and relies on Proposition 2.2.

Lemma 7.2. Let $T^{*}: \mathbb{H} \rightarrow \ell_{2}(I)$ be the analysis operator for a frame $\left\{f_{i}\right\}_{i \in I}$ for $\mathbb{H}$ and let $P$ be the orthogonal projection of $\ell_{2}(I)$ onto $\mathbb{H}$. Then $\left\{P e_{i}\right\}_{i \in I}$ is a Parseval frame for $T^{*}(\mathbb{H})$ which is isomorphic to $\left\{f_{i}\right\}_{i \in I}$.

Proof: Note that $\left\{P e_{i}\right\}_{i \in I}$ is a Parseval frame (Theorem 2.4) with synthesis operator $P$ and analysis operator $T_{1}^{*}$ satisfying $T_{1}^{*}(\mathbb{H})=P\left(\ell_{2}(I)\right)=T^{*}(\mathbb{H})$. By Proposition 2.2, $\left\{P e_{i}\right\}_{i \in I}$ is equivalent to $\left\{f_{i}\right\}_{i \in I}$.

Now we will relate large subspaces of a Hilbert space with the range of the analysis operator of some bounded frame. We also give a quantative version of the result for later use.

Proposition 7.3. Let $\mathbb{H}$ be a subspace of $\ell_{2}(I)$.

(I) The following are equivalent:

(1) The subspace $H$ is large.

(2) The subspace $H$ is the range of the analysis operator of some bounded frame.

(II) The following are equivalent:

(3) The subspace $\mathbb{H}$ is A-large.

(4) If $P$ is the orthogonal projection of $\ell_{2}(I)$ onto $\mathbb{H}$ then $\left\|P e_{i}\right\| \geq A$, for all $i \in I$. 
Proof: (I) $(1) \Rightarrow(2)$ : Suppose $\mathbb{H}$ is large. So, there exists an $A>0$ such that for each $i \in I$, there exists a vector $f_{i} \in \mathbb{H}$ with $\left\|f_{i}\right\|=1$ and $\left|f_{i}(i)\right| \geq A$. Given the projection $P$ of $(2)$ we have

$$
A \leq\left|f_{i}(i)\right|=\left|\left\langle e_{i}, f_{i}\right\rangle\right|=\left|\left\langle P e_{i}, f_{i}\right\rangle\right| \leq\left\|P e_{i}\right\|\left\|f_{i}\right\|=\left\|P e_{i}\right\| .
$$

Note that this also proves (II) $(3) \Rightarrow(4)$.

$(2) \Rightarrow(1)$ : Assume $\left\{f_{i}\right\}_{i \in I}$ is a bounded frame for a Hilbert space $\mathbb{K}$ with analysis operator $T^{*}$ and $T^{*}(\mathbb{K})=\mathbb{H}$. Now, $\left\{P e_{i}\right\}_{i \in I}$ is a Parseval frame for $\mathbb{H}$ which is the range of its own analysis operator. Hence, $\left\{f_{i}\right\}_{i \in I}$ is equivalent to $\left\{P e_{i}\right\}_{i \in I}$ by Proposition 2.2. Since $\left\{f_{i}\right\}_{i \in I}$ is bounded, so is $\left\{P e_{i}\right\}_{i \in I}$. Choose $A>0$ so that $A \leq\left\|P e_{i}\right\| \leq 1$, for all $i \in I$. Then

$$
A \leq\left|\left\langle P e_{i}, P e_{i}\right\rangle\right|=\left|\left\langle P e_{i}, e_{i}\right\rangle\right|=\left|P e_{i}(i)\right| \text {. }
$$

So $\mathbb{H}$ is a large subspace.

Note that this also proves (II) (4) $\Rightarrow(3)$.

Now we need to learn how to decompose the range of the analysis operator of our frames.

Definition 7.4. A closed subspace $\mathbb{H}$ of $\ell_{2}(I)$ is $\mathbf{r}$-decomposable if for some natural number $r$ there exists a partition $\left\{S_{j}\right\}_{j=1}^{r}$ of $I$ so that $P_{S_{j}}(\mathbb{H})=\ell_{2}\left(S_{j}\right)$, for all $j=1,2, \cdots, r$. The subspace $\mathbb{H}$ is finitely decomposable if it is $r$ decomposable for some $r$.

For the next proposition we need a small observation.

Lemma 7.5. Let $\left\{f_{i}\right\}_{i \in I}$ be a Bessel sequence in $\mathbb{H}$ having synthesis operator $T$ and analysis operator $T^{*}$. Let $E \subset I$, and let $\left\{f_{i}\right\}_{i \in E}$ have analysis operator $\left(\left.T\right|_{E}\right)^{*}$. Then

$$
P_{E} T^{*}=\left(\left.T\right|_{E}\right)^{*}
$$

Proof: For all $f \in \mathbb{H}$,

$$
P_{E} T^{*}(f)=P_{E}\left(\sum_{i \in I}\left\langle f, f_{i}\right\rangle e_{i}\right)=\sum_{i \in E}\left\langle f, f_{i}\right\rangle e_{i}=\left(\left.T\right|_{E}\right)^{*}(f) .
$$

We now have

Proposition 7.6. If $\left\{f_{i}\right\}_{i \in I}$ is a unit norm frame for $\mathbb{K}$ with analysis operator $T^{*}$, then for any $0<\epsilon<1, T^{*}(\mathbb{K})$ is r-decomposable for

$$
r=\left(\frac{6\left(\|T\|^{2}+1\right)}{\epsilon}\right)^{4}
$$

(with power 8 in the complex case). 
Proof: We can partition $I$ into $\left\{S_{j}\right\}_{j=1}^{r}$ so that each $\left\{f_{i}\right\}_{i \in S_{j}}$ is a Riesz basic sequence where

$$
r=\left(\frac{6\left(\|T\|^{2}+1\right)}{\epsilon}\right)^{2}
$$

for any $0<\epsilon<1$. Thus, (see the discussion after Theorem 2.1) $\left(\left.T\right|_{S_{j}}\right)^{*}$ is onto for every $j=1,2, \cdots, r$ and hence (by Lemma 7.5) $P_{S_{j}} T^{*}$ is onto for all $j=1,2, \cdots, r$.

Now we can put this altogether.

Theorem 7.7. For every $0<A<1$ and $0<\epsilon<1$, there is a natural number

$$
r=\left(\frac{6\left(A^{2}+1\right)}{\epsilon A^{2}}\right)^{4},
$$

(power 8 in the complex case) so that every A-large subspace of $\ell_{2}(I)$ is $r$ decomposable.

Proof. By Proposition 7.3, if $P$ is the orthogonal projection of $\ell_{2}(I)$ onto $H$, then $\left\|P e_{i}\right\| \geq A$, for all $i \in I$. Then

$$
\left\{f_{i}\right\}_{i \in I}=\left\{\frac{P e_{i}}{\left\|P e_{i}\right\|}\right\}_{i \in I},
$$

is a unit norm frame with Bessel bound $1 / A^{2}$. So by Proposition 7.6, our subspace is $r$-decomposable for

$$
r=\left(\frac{6\left(A^{2}+1\right)}{\epsilon A^{2}}\right)^{4},
$$

for any $0<\epsilon<1$.

\section{Open Problems}

There are a number of important open problems which remain even after the work of [40].

Problem 8.1. Can the $\eta$ and $\theta$ in Theorem 3.2 be improved?

Problem 8.2. Can the values of $r$ in the various results be improved?

It has been shown [14, 23] that every unit norm two tight frame can be partitioned into two linearly independent sets. But, there do not exist universal constants $A, B$ so that all such frames can be partitioned into two subsets each with Riesz bounds $A, B$ [17, 18]. This result raises a fundamental problem.

Problem 8.3. Can every unit norm two tight frame be partitioned into three subsets each of which are Riesz basic sequences with Riesz bounds independent of the dimension of the space? 
Perhaps the most important open problem:

Problem 8.4. Find an implementable algorithm for proving the Paving Conjecture.

The most important case is really finding an algorithm for proving the Feichtinger Conjecture. The Feichtinger Conjecture potentially could have serious applications if we could quickly compute the appropriate subsets which are Riesz basic sequences.

\section{ACKNOWLEDGEMENT}

For many years the Kadison-Singer Problem was a major motivating force for many of us. It's challenges made every day an exciting event. It also brought together mathematicians from many diverse areas of research - especially as the "polynomial people" came in to give the solution. As we discovered more elementary formulations of the problem, it became clear that this problem represented a fundamental idea for finite dimensional Hilbert spaces which was not understood at all. This just made the problem even more interesting. Almost everyone believed that the problem had a negative answer - which probably contributed to the problem remaining open for so long since we were only looking for a counter-example. The solution to this problem by Marcus/Spielman/Srivastave was a major achievement of our time and earned them the Polya Prize, a trip to the International Congress of Mathematicians and recognition yet to be established.

The 54 year search for a solution to the Kadison-Singer Problem represented a large number of papers by many brilliant mathematicians which culminated in the solution to the problem by Marcus/Spielman/Srivastave. We enclose here a brief summary of the historical development of the Kadison-Singer Problem from the direction of the Paving Conjecture. Since the authors are not experts in $C^{*}$-Algebras, we have chosen not to trace the history of the problem from that direction. So we will start in 1979 with the introduction of the Anderson Paving Conjecture. Also, there are hundreds of papers here so we will just consider those papers which introduced new directions (equivalences) of the Paving Conjecture.

- 34] (1959) Kadison and Singer formulate the Kadison-Singer Problem.

- 2] (1979) Anderson reformulates the Kadison-Singer Problem into the Anderson Paving Conjecture. This was significant because it changed the Kadison-Singer Problem from being a specialized problem hidden in $C^{*}$-Algebras and opened it up to everyone in Analysis.

- [8, 9, 29, 30] (1986) Berman/Halpern/Kaftal/Weiss make a deep study of the Paving Conjecture for Laurant Operators. They introduced 
the notion of uniform pavability and they show that matrices with positive coefficients are pavable. They also give a positive solution for paving for the Schatten $C_{p}$-norms for $p=4,6$.

- [10] (1989) Bourgain-Tzafriri prove the famous Restricted Invertibility Theorem which naturally leads to the Bourgain-Tzafriri Conjecture.

- 11] (1991) Bourgain-Tzafriri show that matrices with small entries are pavable.

- [1] (1991) Akemann and Anderson formulate the Akemann-Anderson Projection Paving Conjecture and show it implies a positive solution to the Kadison-Singer Problem. This was important since it reduced paving to paving for a much smaller class of operators - projections.

- 27](2003) Grochenig shows that localized frames satisfy the Feichtinger Conjecture. This conjecture was formulated in [14] but appeared much later.

- [46, 47] (2003-2004) Weaver formulates the Weaver Conjectures and gives a counter-example to a conjecture of Akemann and Anderson which would have implied a positive solution to the Kadison-Singer Problem.

- 14 (2005) Casazza/Christensen/Lindner/Vershynan introduce the Feichtinger Conjecture and show it is equivalent to the BourgainTzafriri Conjecture.

- 12 (2006) Bownik/Speegle make a detailed study of the Feichtinger Conjecture for Wavlet Frames, Gabor Frames and Frames of Translates and relate the Feichtinger Conjecture to Gowers' work on a generalization of Van der Waerdan's Theorem.

- 23, 19] (2006) Casazza/Tremain and Casazza/Fickus/Tremain/Weber show that the Kadison-Singer Problem is equivalent to the Feichtinger Conjecture, the Bourgain-Tzafriri Conjecture, the $R_{\epsilon}$-Conjecture, the Casazza/Tremain Conjecture, and a number of conjectures in Time-Frequency Analysis, Frames of Translates and Hilbert Space Frame Theory.

- 43] (2008) Paulsen and Raghupathi show that paving (respectively, paving Toeplitz operators) is equivalent to paving upper trianguar matrices (respectively, paving upper triangular Toeplitz operators).

- [17] (2009) Casazza/Edidin/Kalra/Paulsen show that paving projections with constant diagonal $1 / 2$ is equivalent to the Paving Conjecture. This is a new direction for paving projections as all previous work involved paving projections with very small diagonals. They also show that the Paving Conjecture fails for 2-paving. 
- 38] (see also [42]) (2010) Lawton and Paulsen independently showed that if the Feichtinger Conjecture holds for Fourier frames, then each set in the partition into Riesz basic sequences can be chosen to be a syndetic set. Paulsen first presented this at GPOTS (2008).

- (2007 - 2010) A large number of papers on the Feichtinger Conjecture appeared. Too numerous to list here. See [36] for a somewhat complete list - especially for reproducing kernel Hilbert spaces and for classical spaces.

- 18] (2011) Casazza/Fickus/Mixon/Tremain give concrete constructions of non-2-pavable projections.

- 13] (2012) Casazza introduces the Sundberg Problem which is implied by the Paving Conjecture.

- 40] (2013) Marcus/Spielman/Srivastava surprise the mathematical community by giving a positive solution to the Kadison-Singer Problem.

Remark 9.1. We were recently made aware of the thesis [39] of Y. Lonke from 1993 which has a proof that BT is equivalent to KS. Since it was written Hebrew, it seems to have been overlooked. We now have English translations [39].

\section{REFERENCES}

[1] C.A. Akemann and J. Anderson, Lyapunov theorems for operator algebras, Mem. AMS 94 (1991).

[2] J. Anderson, Restrictions and representations of states on $C^{*}$-algebras, Trans. AMS 249 (1979) 303-329.

[3] J. Anderson, Extreme points in sets of positive linear maps on B(HI), Jour. Functional Analysis 31 (1979) 195-217.

[4] J. Anderson, A conjecture concerning pure states on $B(\mathbb{H})$ and a related theorem, in Topics in modern operator theory, Birkhäuser (1981) 27-43.

[5] R. Balan, P.G. Casazza, C. Heil and Z. Landau, Density, overcompleteness and localization of frames. I. Theory, J. FOurier Analysis and Appls. 12 (2006) 105 143.

[6] R. Balan, P.G. Casazza, C. Heil and Z. Landau, Density, overcompleteness and localization of frames. II. Gabor systems, J. Fourier Analysis and Appls. 12 (2006) 309 - 344.

[7] A. Baranov and K. Dyakonov, The Feichtinger Conjecture for reproducing kernels in model subspaces, Journal of Geometric Analysis, 21 No. 2 (2011) 276-287.

[8] K. Berman, H. Halpern, V. Kaftal and G. Weiss, Matrix norm inequalities and the relative Dixmier property, Integ. Eqns. and Operator Theory 11 (1988) 28-48.

[9] K. Berman, H. Halpern, V. Kaftal and G. Weiss, Some $C_{4}$ and $C_{6}$ norm inequalities related to the paving problem, Proceedings of Symposia in Pure Math. 51 (1970) 29-41.

[10] J. Bourgain and L. Tzafriri, Invertibility of "large" submatrices and applications to the geometry of Banach spaces and Harmonic Analysis, Israel J. Math. 57 (1987) 137-224. 
[11] J. Bourgain and L. Tzafriri, On a problem of Kadison and Singer, J. Reine Angew. Math. 420 (1991), 1-43.

[12] M. Bownik and D. Speegle, The Feichtinger conjecture for wavelet frames, Gabor frames and frames of translates, Canad. J. Math 58 No. 6 (2006) 1121 - 1143.

[13] P.G. Casazza, The Kadison-Singer and Paulsen Problems in finite frame theory, in Finite Frames: Theory and Applications, P.G. Casazza and G. Kutyniok Eds., Birkhauser, Boston (2013).

[14] P.G. Casazza, O. Christensen, A. Lindner and R. Vershynin, Frames and the Feichtinger conjecture, Proceedings of AMS, 133 No. 4 (2005) 1025-1033.

[15] P.G. Casazza, O. Christensen and N.J. Kalton, Frames of translates, Collect. Math. 52 No. 1 (2001) 35-54.

[16] P.G. Casazza and D. Edidin, Equivalents of the Kadison-Singer Problem, Contemp. Math 435 (2007) 123 - 142.

[17] P.G. Casazza, D. Edidin, D. Kalra and V. Paulsen, Projections and the KadisonSinger Problem, Operators and Matrices, 1(3) (2009) 391-408.

[18] P.G. Casazza, M. Fickus, D. Mixon and J.C. Tremain, The Bourgain-Tzafriri Conjecture and concrete construction of non-pavable projections, Operators and Matrices 5 no. 2 (2011) $353-363$.

[19] P.G. Casazza, M. Fickus, J.C. Tremain and E. Weber, The Kadison-Singer Problem in Mathematics and Engineering: A detailed account, Contemp. Math. 414 Operator Theory, Operator Algebras and Appls. D. Han, P.E.T. Jorgensen and D.R. Larson, Eds. (2006) 297 - 356.

[20] P.G. Casazza and G. Kutyniok, Eds. Finite Frames: Theory and Applications, Birkhauser, Boston (2013).

[21] P.G. Casazza, G. Kutyniok and D. Speegle, A decomposition theorem for frames and the Feichtinger Conjecture, Proceedings of AMS 136 (2008)2043 - 2053.

[22] P.G. Casazza and J.C. Tremain, The Kadison-Singer problem in Mathematics and Engineering, Proceedings of the National Academy of Sciences 103 No. 7 (2006) 2032 - 2039.

[23] P.G. Casazza and J.C. Tremain, Revisiting the Bourgain-Tzafriri Restricted Invertibility Theorem, Operators and Matrices 3 No. 1(2009) 97 - 110.

[24] O. Christensen, An introduction to frames and Riesz bases, Birkhauser, Boston, 2003.

[25] P.A.M. Dirac, Quantum Mechanics, 3rd Ed., Oxford University Press, London (1947).

[26] R.J. Duffin and A.C. Schaeffer, A class of nonharmonic Fourier series, Trans. AMS 72 (1952) 341-366.

[27] K.H. Gröchenig, Localized frames are finite unions of Riesz sequences, Adv. Comp. Math. 18 (2003) 149-157.

[28] H. Halpern, V. Kaftal and G. Weiss, The relative Dixmier property in discrete crossed products, J. Funct. Anal. 69 (1986) 121-140.

[29] H. Halpern, V. Kaftal and G. Weiss, Matrix pavings and Laurent operators, J. Op. Th. 16 (1986) 121-140.

[30] H. Halpern, V. Kaftal and G. Weiss, Matrix pavings in B(H), Proc. $10^{\text {th }}$ International conference on operator theory, Increst 1985; Advances and Applications 24 (1987) 201-214.

[31] D. Han, K. Kornelson, D. Larson, and E. Weber, Frames for Undergraduates, Student Mathematical Library, AMS 40 (2007). 
[32] D. Han and D.R. Larson, Frames, bases and group representations, Memoirs AMS 697 (2000).

[33] P. Jaming, Inversibilité restreinte, problem d'extension de Kadison-Singer et applications a l'analyse harmonique, Preprint.

[34] R. Kadison and I. Singer, Extensions of pure states, American Jour. Math. 81 (1959), $383-400$.

[35] B. Kashin, L. Tzafriri, Some remarks on the restrictions of operators to coordinate subspaces, unpublished.

[36] S. Lata, The Feichtinger Conjecture and reproducing kernel Hilbert spaces, PhD Thesis, University of Houston (2010).

[37] S. Lata and V. Paulsen, The Feichtinger Conjecture and Reproducing Kernel Hilbert Spaces, Indiana Univ. Math J. 60 No.4 (2011) 1303 - 1317.

[38] W. Lawton, Minimal sequences and the Kadison-Singer Problem, Bulletin Malaysian Math Soc. (Second Series) 33 No. 2 (2010) 169 - 176.

[39] Y. Lonke, Extensions of pure states and the Laurant operator, MS Thesis, University of Jerusalem, Thesis director: Lior Tzafriri (1993). (See www.framerc.org for an English translation).

[40] A. Marcus, D. Spielman and N. Srivastave, Interlacing families II: Mixed Characteristic Polynomials and the Kadison-Singer Problem, arXiv 1306.3969v4.

[41] V. Paulsen, A dynamical systems approach to the Kadison-Singer Problem, J. Functional Analysis 255 (2008) 120 - 132.

[42] V. Paulsen, Syndetic sets, paving and the Feichtinger Conjecture, Proceedings AMS 139 No. 3 (2011) 1115 - 1120.

[43] V.I. Paulsen and M. Raghupathi, Some new equivalences of Anderson's paving conjecture, Proc. AMS 136 (2008) 4275 - 4282.

[44] S. Popa, $A \Pi_{1}$ factor approach to the Kadison-Singer Problem, arXiv: 1303.1424.

[45] D. Spielman and N. Srivastave, An elementary proof of the Restricted Invertibility Theorem, Israel Journal of Math. 190 (2012)

[46] N. Weaver, The Kadison-Singer Problem in discrepancy theory, Discrete Math. 278 (2004), 227-239.

[47] N. Weaver, A counterexample to a conjecture of Akemann and Anderson, Bulletin of the London Math Society, 35 No. 1 (2003) 65 - 71..

[48] R.M. Young, An introduction to nonharmonic Fourier series, Academic Press, New York (1980).

Department of Mathematics, University of Missouri-Columbia, Columbia, MO 65211

E-mail address: casazzap@missouri.edu; tremainjc@missouri.edu 